\title{
Auswertung der Betriebsdaten von Erdwärmesonden und Ableitung des Wärmeleitfähigkeitskatasters Oberösterreich
}

Zusammenfassung: Die Betriebsdaten von 28 EWS-Anlagen des Winters 2008/09 wurden durch das Amt der OÖ Landesregierung mit der MS-Excel ${ }^{\mathrm{TM}}$ Rechentabelle des ÖWAV ausgewertet. Die daraus abgeleiteten Wärmeleitfähigkeiten des Untergrunds wurden gemeinsam mit Daten aus Thermal Response Tests in einem Wärmeleitfähigkeitskataster veröffentlicht. Zudem wurden die oberflächennahen Wärmeleitfähigkeiten der OÖ Gesteine systematisch gemessen und Literaturwerten gegenübergestellt.

Aus Anlass der Herausgabe der SIA $384 / 6$ wurden die Jahresmittelwerte der Lufttemperaturen und der Bodenoberflächentemperaturen in Österreich den Schweizer Klimadaten gegenübergestellt.

Interpretation of operational data from geothermal boreholes, serving as a basis for preparing a heat conductivity register for Upper Austria

Summary: The provincial government of Upper Austria has analysed the operational data of 28 geothermal borehole systems collected in the winter of 2008-09 using the ÖWAV's (Austrian Water and Waste Management Association's) MS-Excel ${ }^{\mathrm{TM}}$ ready reckoner. The heat conductivity levels of the ground obtained were published together with data from thermal response tests in a heat conductivity register. In addition, near-surface heat conductivity values of Upper Austrian rocks were measured systematically and compared with data from the relevant literature.

On the occasion of the publication of the Swiss SIA 384/6 standard, the mean annual air and ground-surface temperatures in Austria were compared with the Swiss climate data.

1. Bemessung von Erdwärmesonden nach ÖWAV Regelblatt 207

EWS werden oft in Gebieten errichtet, wo mehrere Grundwasser-Stockwerke mit unterschiedlichem Grundwasserchemismus und/oder unterschiedlichen Druckverhält-

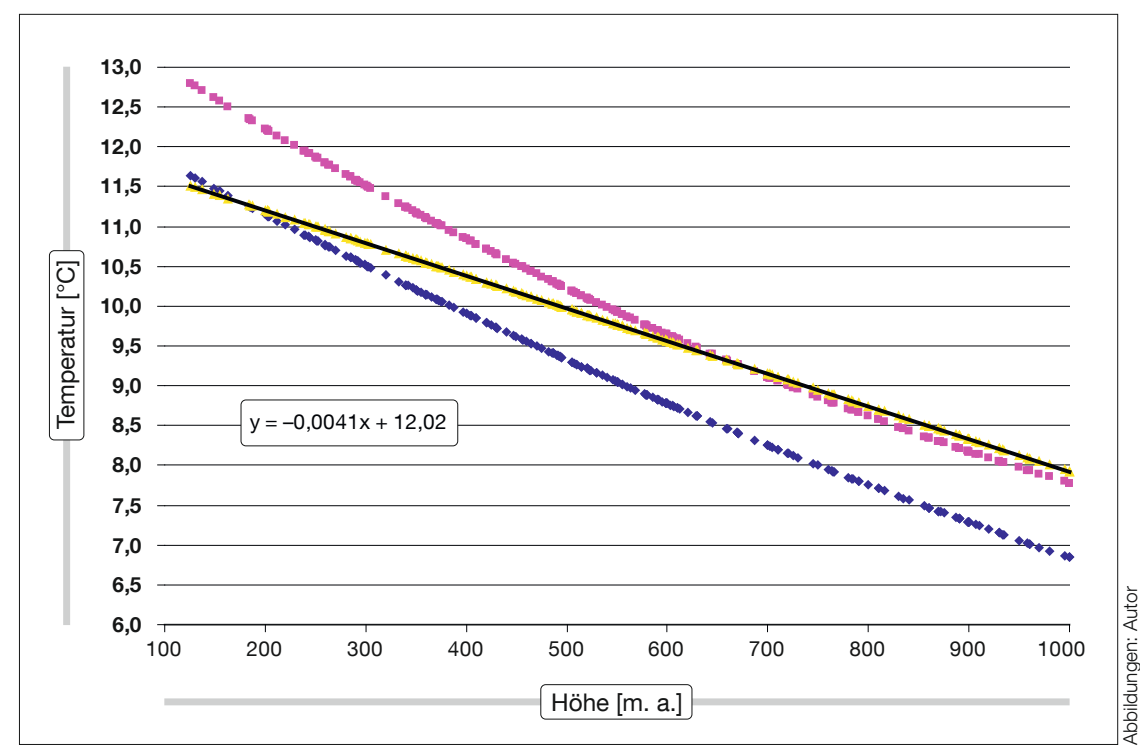

Abb. 1: Regressionsgerade der mittleren österreichischen Bodentemperaturen in $20 \mathrm{~cm}$ Tiefe abzüglich 0,5 K, verglichen mit den Regressionskurven Alpennordseite (blau) und Alpensüdseite (rosa) der SIA 384/6.

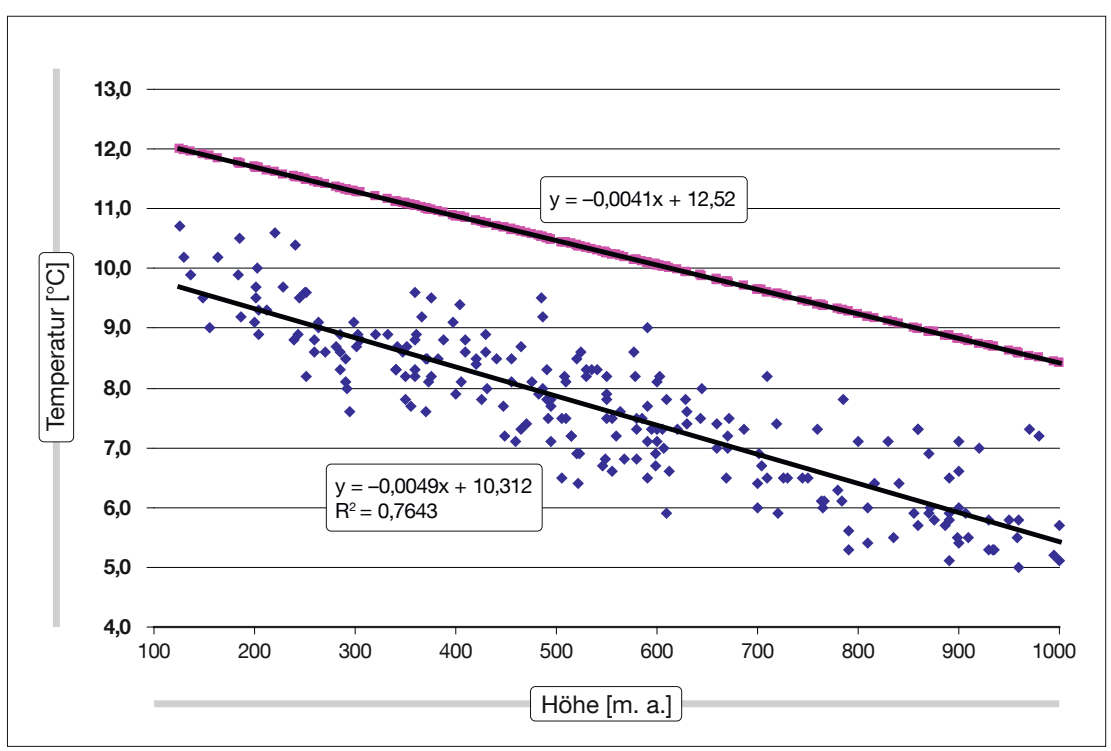

Abb. 2: Jahresmittelwerte der Lufttemperaturen in Österreich bis 1000 m ü.A., verglichen mit Regressionsgerade der mittleren Bodenoberflächentemperaturen.

nissen vorliegen. Um nachteilige Auswirkungen auf das Grundwasser zu vermeiden, ist die Verbindung unterschiedlicher Grundwasser-Stockwerke während des Bohrvorgangs möglichst gering zu halten und durch die Sondenverpressung dauerhaft zu unterbinden. EWS sind deshalb so ausreichend zu bemessen, dass die erforderlichen Leistungen und Energiemengen für Heizen und Kühlen über die gesamte Lebensdauer der Anlage bereitgestellt werden können, ohne dass die Sondenumgebung unter den Gefrierpunkt abgekühlt und unzulässig erwärmt wird (erstes Ele- 
TABELLE 1

Ergebnisse von Thermal Response-Tests in Österreich

\begin{tabular}{|c|c|c|c|c|c|}
\hline Ort & $\begin{array}{l}\text { Länge EWS } \\
\text { [m] }\end{array}$ & $\begin{array}{l}\text { Wärmeleit- } \\
\text { fähigkeit } \\
{[\mathrm{W} / \mathrm{m}, \mathrm{K}]}\end{array}$ & $\begin{array}{l}\text { spez. Wärme- } \\
\text { kapazität } \\
{\left[\mathrm{MJ}^{3} \mathrm{~m}^{3}, \mathrm{~K}\right]}\end{array}$ & $\begin{array}{l}\text { mittlere Unter- } \\
\text { grundtemperatur } \\
{\left[{ }^{\circ} \mathrm{C}\right]}\end{array}$ & Untergrundaufbau \\
\hline Klagenfurt & 80,5 & 3,30 & & 12,20 & Grauwacke (Phyllite), kein GW \\
\hline Peuerbach & 159 & 2,70 & 2,10 & 11,90 & $90 \mathrm{~m}$ Quarzsande, $30 \mathrm{~m}$ Molasse \\
\hline Thalheim & 200 & 2,40 & 2,20 & 12,50 & $\begin{array}{l}12 \mathrm{~m} \text { Kies, ab } 6 \mathrm{~m} \text { GW, ab 12m } \\
\text { Molasse }\end{array}$ \\
\hline Thalheim & 150 & 2,30 & 2,20 & 12,50 & $\begin{array}{l}12 \mathrm{~m} \text { Kies, ab } 6 \mathrm{~m} \mathrm{GW} \text {, ab 12m } \\
\text { Molasse }\end{array}$ \\
\hline Wels & 100 & 2,70 & 2,40 & 11,60 & $\begin{array}{l}12 \mathrm{~m} \text { Kies, ab } 6 \mathrm{~m} \mathrm{GW} \text {, ab 12m } \\
\text { Molasse }\end{array}$ \\
\hline Villach & 137 & 2,20 & 2,20 & 12,00 & $\begin{array}{l}70 \mathrm{~m} \text { Grobsand-Kies, ab } 9 \mathrm{~m} \text { GW, bis } \\
139 \mathrm{~m} \text { Seeton }\end{array}$ \\
\hline Lannach & 97 & 2,21 & & 13,42 & Kies- und Tonabfolgen \\
\hline Wien 19 & 150 & 2,14 & & 10,20 & Ton, Sandsteinabfolgen \\
\hline Graz Raaba & 100 & 2,19 & & 13,40 & Kies, Ton, Sandabfolgen \\
\hline Feldkirch & & 2,60 & & 12,20 & \\
\hline Brandnertal & & 4,62 & & 7,50 & \\
\hline Lustenau & & 1,93 & & 11,80 & \\
\hline
\end{tabular}

ment der doppelten Sicherheit). Dies ist gegeben, wenn die mittlere Temperatur des Wärmeträgermediums (Mittelwert EWS Ausgangs- und EWS Eingangstemperatur) nach Erreichen eines neuen Gleichgewichtszustandes im Lastfall Heizen $-1,5^{\circ} \mathrm{C}$ nicht unterschreitet und im Lastfall Kühlen die max. Temperatur des Wärmeträgermediums $30^{\circ} \mathrm{C}$ nicht überschreitet. Wird die maximal zulässige Entzugsleistung bzw. die maximal zulässige Jahresentzugsarbeit überschritten, ist mit einer Unterkühlung und mit häufigen Frost-Tau-Wechseln um die Sonden zu rechnen. Dadurch kann die Dichtheit der Sondenverpressung beeinträchtigt und die Sondenrohre können beschädigt werden. Weiters ist mit einer wesentlichen Verringerung des Wirkungsgrads der Wärmepumpe zu rechnen.

Als zweites Element der doppelten Sicherheit dürfen nur Verpresssuspensionen verwendet werden, deren Eignung und Frost-Tau-Wechselbeständigkeit nachgewiesen sind. Die Durchführung der Prüfung der Frost-Tau-Wechselbeständigkeit hat in Anlehnung an die Studie „Eignungsuntersuchung von Verpressmaterialien für Erdwärmesonden" zu erfolgen (Niederbrucker, Steinbacher 2007).

Im ÖWAV-Arbeitsausschuss wurde intensiv sowohl unter dem Aspekt des vorsorgenden Grundwasserschutzes als auch unter dem Aspekt der wirtschaftlichen Anlagenerrichtung über die „richtige“ Bemessung von EWS diskutiert. Letztlich wurden folgende vier Bemessungsverfahren in das Regelblatt aufgenommen.
1.1. Bemessung nach Betriebsdaten bestehender Anlagen

Diese wurde aufgrund der Erfahrungen aus zahlreichen Bestandsanlagen an die erste Stelle gereiht aufgenommen, wohl wissend, dass große Defizite bei der Dokumentation der Betriebsdaten bestehen. EWS können bei vergleichbaren Standortbedingungen mit den spezifischen Ent- zugsleistungen von bestehenden Anlagen bemessen werden, wenn durch nachvollziehbare Betriebsdaten nachgewiesen ist, dass die Grenzen der Temperatur des Wärmeträgermediums eingehalten werden. Die Betriebsdaten sollen über einen Zeitraum von 2 Jahren vorliegen und für jede Heizperiode umfassen: Jahresbetriebsstunden Heizen, wöchentliche Aufzeichnungen der min. Vorlauf- und Rück-

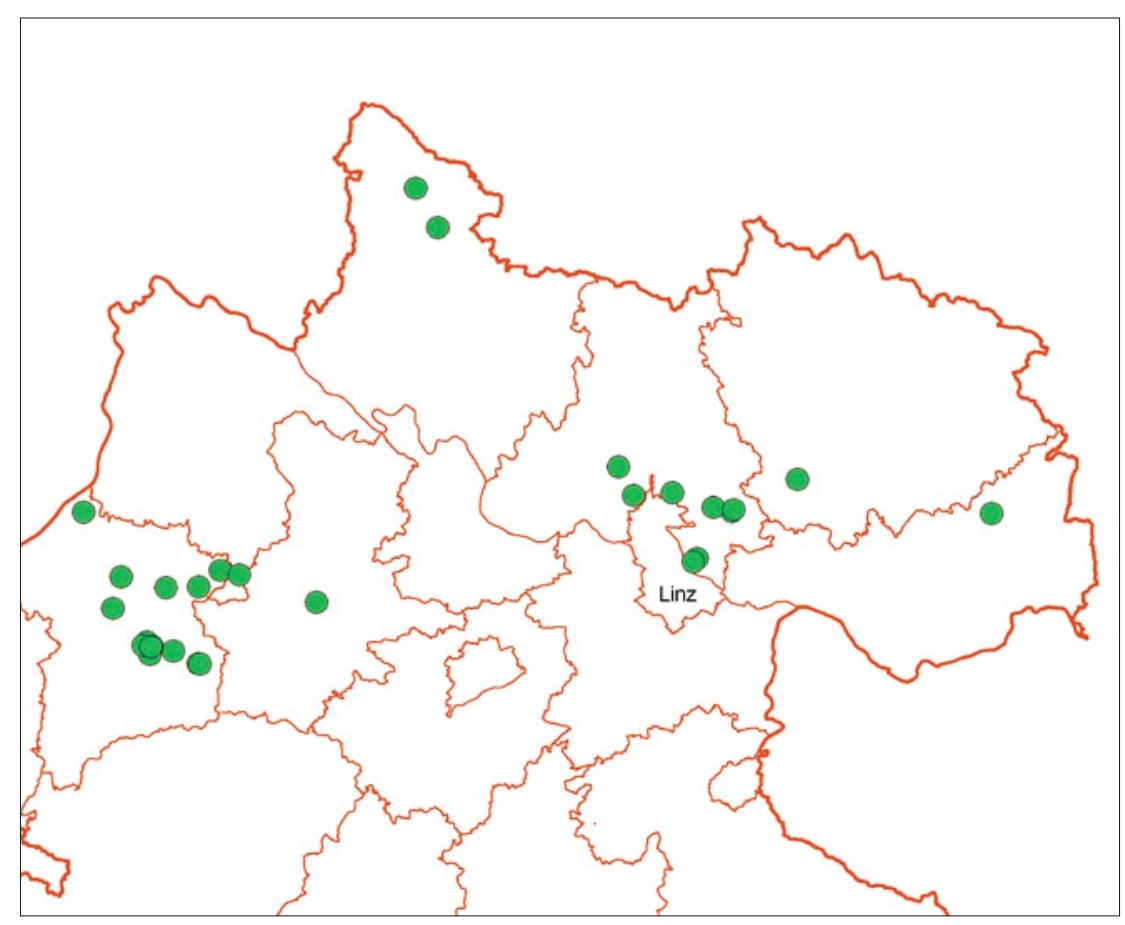

Abb. 3: Lage der ausgewerteten EWS in nördlichen und westlichen OÖ Bezirken. 


\begin{tabular}{|c|c|c|c|c|c|c|}
\hline & \multirow{2}{*}{\multicolumn{2}{|c|}{ Gesteinstyp }} & \multicolumn{2}{|c|}{ Wärmeleitfähigkeit $[\lambda], W /(m . K)$} & \multirow{2}{*}{$\begin{array}{l}\text { spez. Wärme- } \\
\text { kapazität [c] } \\
\mathrm{MH} /\left(\mathbf{m}^{3} . \mathrm{K}\right)\end{array}$} & \multirow{2}{*}{$\begin{array}{l}\text { Dichte [p] } \\
103 \mathrm{~kg} / \mathrm{m3}\end{array}$} \\
\hline & & & & $\begin{array}{l}\text { empf. } \\
\text { Rechenwert }\end{array}$ & & \\
\hline \multirow{6}{*}{ Lockergesteine } & \multicolumn{2}{|l|}{ Ton/Schluff, trocken } & $0,4-1,0$ & 0,5 & $1,5-1,6$ & $1,8-2,0$ \\
\hline & \multicolumn{2}{|c|}{ Ton/Schluff, wassergesättigt } & $0,9-2,3$ & 1,7 & $2,0-2,8$ & $2,0-2,2$ \\
\hline & \multicolumn{2}{|l|}{ Sand, trocken } & $0,3-0,8$ & 0,4 & $1,3-1,6$ & $1,8-2,2$ \\
\hline & \multicolumn{2}{|c|}{ Sand, wassergesättigt } & $1,5-4,0$ & 2,4 & $2,2-2,8$ & $1,9-2,3$ \\
\hline & \multicolumn{2}{|l|}{ Kies/Steine, trocken } & $0,4-0,5$ & 0,4 & $1,3-1,6$ & $1,8-2,2$ \\
\hline & \multicolumn{2}{|c|}{ Kies/Steine, wassergesättigt } & $1,6-2,0$ & 1,8 & $2,2-2,6$ & $1,9-2,3$ \\
\hline \multirow{10}{*}{$\begin{array}{l}\text { Sedimentäre } \\
\text { Festgesteine }\end{array}$} & \multicolumn{2}{|l|}{ Ton-/Schluffstein } & $1,1-3,5$ & 2,2 & $2,1-2,4$ & $2,4-2,6$ \\
\hline & \multicolumn{2}{|l|}{ Sandstein } & $1,3-5,1$ & 2,3 & $1,8-2,6$ & $2,2-2,7$ \\
\hline & \multicolumn{2}{|l|}{ Konglomerat/Brekzie } & $1,3-5,1$ & 2,3 & $1,8-2,6$ & $2,2-2,7$ \\
\hline & \multicolumn{2}{|l|}{ Mergelstein } & $1,5-3,5$ & 2,1 & $2,2-2,3$ & $2,3-2,6$ \\
\hline & \multicolumn{2}{|l|}{ Kalkstein } & $2,5-4,0$ & 2,8 & $2,1-2,4$ & $2,4-2,7$ \\
\hline & \multicolumn{2}{|l|}{ Dolomitstein } & $2,8-4,3$ & 3,2 & $2,1-2,4$ & $2,4-2,7$ \\
\hline & \multicolumn{2}{|c|}{ Sulfatgestein (Anhydrit) } & $1,5-7,7$ & 4,1 & 2,0 & \\
\hline & \multicolumn{2}{|l|}{ Sulfatgestein (Gips) } & $1,3-2,8$ & 1,6 & 2,0 & \\
\hline & \multicolumn{2}{|c|}{ Chloridgestein (Stein-/Kalisalz) } & $5,3-6,4$ & 5,4 & 1,2 & $2,1-2,2$ \\
\hline & \multicolumn{2}{|l|}{ Steinkohle } & $0,3-0,6$ & 0,4 & $1,3-1,8$ & \\
\hline \multirow{8}{*}{$\begin{array}{l}\text { Magmatische } \\
\text { Festgesteine }\end{array}$} & \multicolumn{2}{|l|}{ Tuffit } & 1,1 & 1,1 & & \\
\hline & \multirow{2}{*}{$\begin{array}{l}\text { Vulkanit, sauer bis } \\
\text { intermediär }\end{array}$} & z.B. Rhyolit, Trachyt & $3,1-3,4$ & 3,3 & 2,1 & 2,6 \\
\hline & & z. B. Latit, Dacit & $2,0-2,9$ & 2,6 & 2,9 & $2,9-3,0$ \\
\hline & $\begin{array}{l}\text { Vulkanit, basisch } \\
\text { bis ultrabasisch }\end{array}$ & z.B. Andesit, Basalt & $1,3-2,3$ & 1,7 & $2,3-2,6$ & $2,6-3,2$ \\
\hline & Plutonit, sauer bis & Granit & $2,1-4,1$ & 3,4 & $2,1-3,0$ & $2,4-3,0$ \\
\hline & intermediär & Syenit & $1,7-3,5$ & 2,6 & 2,4 & $2,5-3,0$ \\
\hline & Plutonit, basisch & Diorit & $2,0-2,9$ & 2,6 & 2,9 & $2,9-3,0$ \\
\hline & bis ultrabasisch & Gabbro & $1,7-2,5$ & 1,9 & 2,6 & $2,8-3,1$ \\
\hline & gering metamornh & Tonschiefer & $1,5-2,6$ & 2,1 & $2,2-2,5$ & $2,4-2,7$ \\
\hline & 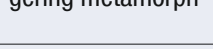 & Kieselschiefer & $4,5-5,0$ & 4,5 & 2,2 & $2,5-2,7$ \\
\hline & & Marmor & $1,3-3,1$ & 2,5 & 2,0 & $2,5-2,8$ \\
\hline Metamorphe & & Quarzit & $5,0-6,0$ & 5,5 & 2,1 & $2,5-2,7$ \\
\hline Festgesteine & mittel bis hoch & Phyllit & $1,5-3,1$ & 2,2 & $2,2-2,4$ & $2,4-2,7$ \\
\hline & metamorph & Glimmerschiefer & $1,5-3,1$ & 2,2 & $2,2-2,4$ & $2,4-2,7$ \\
\hline & & Gneis & $1,9-4,0$ & 2,9 & $1,8-2,4$ & $2,4-2,7$ \\
\hline & & Amphibolit & $2,1-3,6$ & 2,9 & $2,0-2,3$ & $2,6-2,9$ \\
\hline & Bentonit & & $0,5-0,8$ & 0,6 & $\sim 3,9$ & \\
\hline & Beton & & $0,9-2,0$ & 1,6 & $\sim 1,8$ & $\sim 2,0$ \\
\hline & Eis $\left(-10^{\circ} \mathrm{C}\right)$ & & 2,32 & & 1,87 & 0,919 \\
\hline Andere Stoffe & Kunststoff (PE) & & 0,39 & & & \\
\hline & Luft $\left(0-20^{\circ} \mathrm{C}\right)$ & & 0,02 & & 0,0012 & 0,0012 \\
\hline & Stahl & & 60 & & 3,12 & 7,8 \\
\hline & Wasser $\left(+10^{\circ} \mathrm{C}\right)$ & & 0,59 & & 4,15 & 0,999 \\
\hline
\end{tabular}

lauftemperatur des Wärmeträgermediums bei Anlagenbetrieb, Stromverbrauch Wärmepumpe, Stromverbrauch der elektrischen Zusatzheizung bei monoelektri- schen Anlagen, Jahresbetriebsstunden Kühlen. Unterschiedliche Betriebsbedingungen (z. B. Sondenabstände, Jahresbetriebsstunden Heizen und Kühlen) sind gesondert zu berücksichtigen. Eine MSExcel $^{\mathrm{TM}}$ Rechentabelle für die Bemessung von EWS nach SIA 384/6, mit der unterschiedliche Betriebsbedingungen berück- 
TABELLE 3

Wärmeleitfähigkeiten und spezifischen Wärmekapazitäten der Lockergesteine in Oberösterreich

\begin{tabular}{|c|c|c|c|c|c|}
\hline \multirow{2}{*}{$\begin{array}{l}\text { Geologische } \\
\text { Bezeichnung }\end{array}$} & \multirow{2}{*}{$\begin{array}{l}\text { Anzahl } \\
\text { Messungen }\end{array}$} & \multicolumn{2}{|c|}{ Wärmeleitfähigkeit [W/m,K] } & \multicolumn{2}{|c|}{ spez. Wärmekapazität [MJ/m³,K] } \\
\hline & & Bandbreite & Median & Bandbreite & Median \\
\hline Böhmische Masse: Granite, Gneise, Schiefergneise & 14 & $1,56-7,04$ & 2,06 & $1,55-2,19$ & 1,75 \\
\hline Kalkalpen: Kalke, Dolomite, Mergel & 9 & $1,88-4,21$ & 2,60 & $1,82-2,23$ & 1,99 \\
\hline Flysch: Mergel, Sandstein, & 6 & $1,43-3,74$ & 2,42 & $1,47-2,02$ & 1,84 \\
\hline $\begin{array}{l}\text { Sande der Molasse: Linzer , Pleschinger, Atzbacher, } \\
\text { Mehrnbacher, Enzenkirchner }\end{array}$ & 10 & $0,33-2,46$ & 1,49 & $1,46-2,49$ & 2,11 \\
\hline $\begin{array}{l}\text { Schlier der Molasse: Älterer, Robulus, Braunauer, } \\
\text { Haller, Rieder, Vöckla, Ottnanger }\end{array}$ & 14 & $0,79-1,75$ & 1,42 & $1,43-2,32$ & 2,17 \\
\hline Molasse: Neogene Schotter & 2 & $1,46-2,41$ & 1,94 & $1,59-1,91$ & 1,75 \\
\hline $\begin{array}{l}\text { Quartäre Kiese: Austufe, Niederterrasse, Hochterras- } \\
\text { se, Konglomerat, Moränen, Deckenschotter }\end{array}$ & 18 & $0,61-2,74$ & 1,51 & $1,21-2,21$ & 1,73 \\
\hline Quartäre Lösslehmbedeckung & 3 & $1,21-1,61$ & 1,23 & $2,04-2,23$ & 2,06 \\
\hline
\end{tabular}

sichtigt werden können, findet sich auf www.oewav.at im Bereich „Download“.

\subsection{Bemessung nach VDI 4640}

EWS mit Wärmepumpen-Betriebszeiten von 1.800 bis $2.400 \mathrm{~h} / \mathrm{a}$ können bei Betrieb ohne Kühlung mit den spezifischen Entzugsleistungen der Tabelle 7 des ÖWAVRegelblatts 207 bemessen werden. Die Entzugsleistungen dieser Tabelle gelten für Anlagen mit einer Bohrung. Die $\mathrm{Zu}-$ ordnung des Anlagenstandortes zu den spezifischen Entzugsleistungen der einzelnen Gesteine ist jedoch mit großen Unsicherheiten behaftet.

\subsection{Bemessung nach SIA $384 / 6$}

Bei näherer Kenntnis der Wärmeleitfähigkeit des Untergrunds am Sondenstandort kann die Sondenauslegung nach der Schweizer Norm SIA 384/6 „Erdwärmesonden" erfolgen. In dieser Norm sind die Diagramme enthalten, aus denen Eingabeparameter für die MS-Excel ${ }^{\mathrm{TM}}$ Rechentabelle des ÖWAV entnommen werden können.

\subsection{Bemessung von Sondenfeldern mittels numerischer Modellierung}

Sofern am Standort die thermischen Untergrundeigenschaften nicht genau bekannt sind, ist für die Planung von Sondenfeldern eine Pilotbohrung auszuführen und sind die thermischen Untergrundeigenschaften genau zu erkunden (Thermal Response Test, Messung des Temperaturprofils). Die gewonnenen Daten bilden die Grundlage für eine numerische Modellierung. Diese soll den Zeitraum bis zum Erreichen ei- nes neuen thermischen Gleichgewichtszustandes im Untergrund abdecken (in der Regel 5 bis 50 Jahre).

\section{Auswirkungen des Temperatur- regimes im Untergrund auf die Bemessung von EWS}

Die mögliche Entzugsleistung von EWS ist direkt proportional zur Differenz zwischen mittlerer Untergrundtemperatur und mittlerer Auslegungstemperatur (meist $-1,5^{\circ} \mathrm{C}$ ), weshalb deren Ermittlung besondere Bedeutung zukommt.

Die Temperatur der Erdoberfläche wird durch ein Gleichgewicht zwischen einstrahlender Sonnenenergie, Wärmeabstrahlung in das Weltall und dem geothermischen Wärmefluss bestimmt. Infolge der im Verhältnis kleinen Wärmeströme aus dem Erdinneren von etwa 0,05 bis $0,12 \mathrm{~W} / \mathrm{m}^{2}$ ist dieser Anteil an der Energiebilanz der Erdoberfläche sehr gering. Die Sonneneinstrahlung beträgt hingegen bis $\mathrm{zu} 1.000 \mathrm{~W} / \mathrm{m}^{2}$.

Der Einfluss der jahreszeitlich bedingten Temperaturschwankungen reicht abhängig von Bodenaufbau, Höhenlage, Wassergehalt sowie Grundwasserdynamik bis in Tiefen von 10 bis $20 \mathrm{~m}$ (atmosphärische Pufferzone). Erst darunter treten keine jahreszeitlich bedingten Temperaturschwankungen auf.

Die Bodenoberflächentemperatur sinkt mit zunehmender Höhe über dem Meeresspiegel. Es stehen folgende Datenquellen mit abnehmender Genauigkeit zur Verfügung:

- ungestörte Temperaturprofilmessungen in Bohrungen bzw. EWS

- Messungen der ungestörten mittleren Untergrundtemperatur bei Inbetriebnahme von EWS
- Bodenoberflächentemperaturmessstellen der ZAMG, daraus abgeleitet Regressionsgerade mit Höhe über Meer

- Karte über mittlere Bodentemperatur der GBA

- Ableitung aus der mittleren Jahreslufttemperatur

Aus den Datenreihen 1997 bis 2007 von ca. 150 Bodentemperaturmessstellen der Zentralanstalt für Meteorologie und Geodynamik(ZAMG) wurdefür $20 \mathrm{~cm}$ Messtiefe die Regressionsgerade in Abhängigkeit von der Höhe über Meer mit Bestimmtheitsmaß von 0,93 abgeleitet (Götzl 2009). Wegen der Streuung der Messwerte und lokaler Unsicherheiten ist für Heizzwecke $0,5 \mathrm{~K}$ zu subtrahieren, womit sich die Regressionsgerade mit $\mathrm{y}=-0,0041 \mathrm{x}+12,02(A b b .1)$ ergibt. Bei Vorliegen konkreter Messergebnisse (z.B. Messung der unbeeinflussten mittleren Soletemperatur der EWS) sollten diese Messwerte verwendet werden. Der Vergleich mit den Regressionskurven der SIA 384/6, zeigt eine gute Übereinstimmung mit Figur 5 (Alpennordseite) bis $300 \mathrm{~m}$ ü. A. und mit Figur 6 (Alpensüdseite) von 500 bis $1000 \mathrm{~m}$ ü. A.

Für die Normalzahlen des Jahresmittels der Lufttemperaturen von 223 Messstellen < $1000 \mathrm{~m}$ ü. A. (Hydrographisches Jahrbuch von Österreich 2005, 2008) wurde die Regressionsgerade in Abhängigkeit von der Höhe über Meer mit $\mathrm{y}=-0,0049 \mathrm{x}+10,312$ mit Bestimmtheitsmaß von nur $0,76(A b b .2)$ abgeleitet.

Die Bodenoberflächentemperaturen liegen in Österreich im Mittel um 2,6 K über den gemessenen mittleren Jahreslufttemperaturen.

Die Temperatur von der Erdoberfläche zum Erdinnern nimmt im Mittel alle $100 \mathrm{~m}$ 


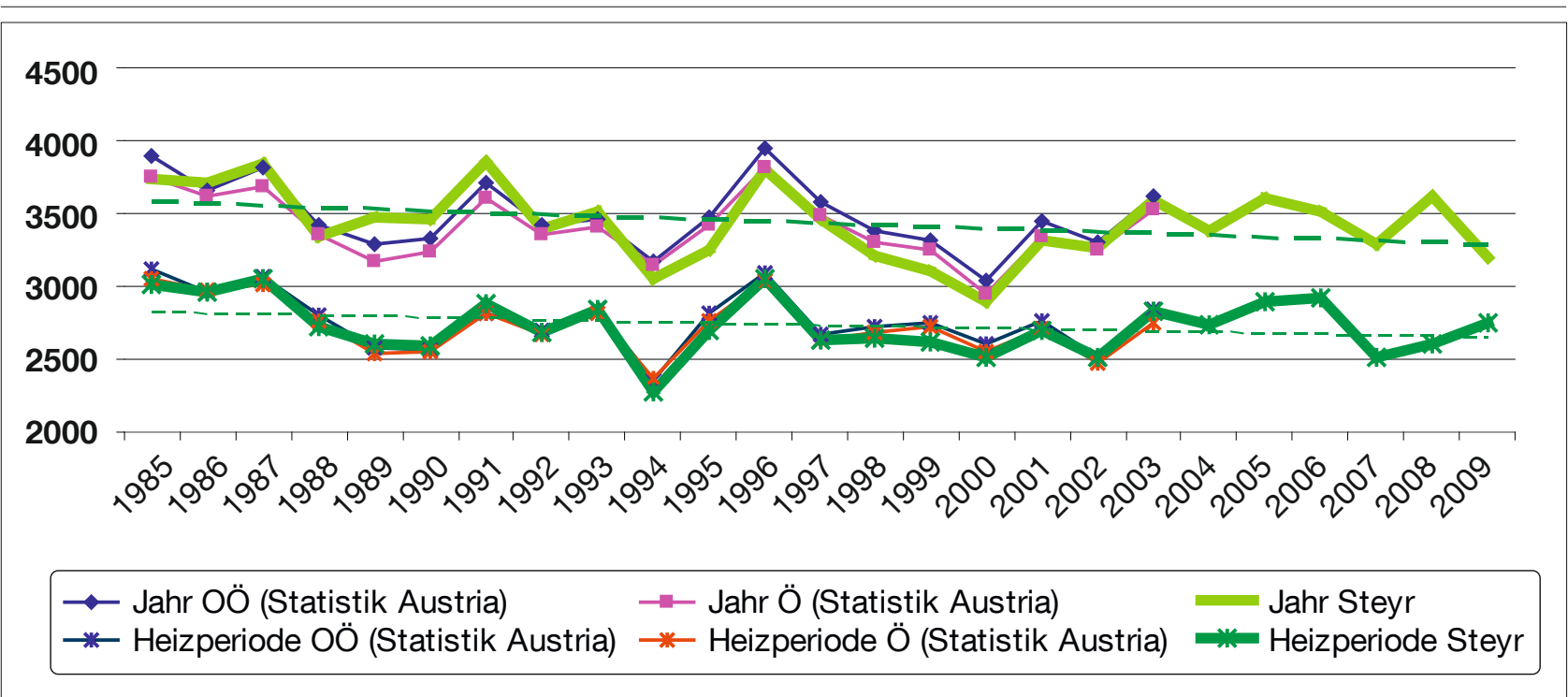

Abb. 4: Langzeitverlauf der Heizgradtage (Überwachungsbericht des oö Luftmessnetzes, Jahresbericht 2007).

um $3 \mathrm{~K}$ zu (geothermischer Gradient). Die kontinuierliche Wärmezunahme ist an Schwächezonen der Erdkruste, wo der Wärmefluss vom Erdinnern stärker ist, größer als in Regionen, wo die Erdkruste mächtiger ist, wie etwa unter den Alpen. In alpinen Lagen sind geothermische Gradienten von $2,5 \mathrm{~K}$ je $100 \mathrm{~m}$ bekannt. Entlang von geologischen Störungszonen und in Exfiltrationsbereichen von Thermalwässern sind geothermische Gradienten von $5 \mathrm{~K}$ je $100 \mathrm{~m}$ bekannt. Es empfiehlt sich, vor Inbetriebnahme von EWS-Anlagen die ungestörte mittlere Untergrundtemperatur zu messen und zu dokumentieren, um für die Planung neuer Anlagen genauere Kenntnisse über die Bodenoberflächentemperatur und den thermischen Gradienten zu erlangen.

\section{Literaturdaten der Wärmeleit-} fähigkeit und spezifischen Wärmekapazität des Untergrunds

Die thermischen Eigenschaften des Untergrunds werden von der Wärmeleitfähigkeit und von der spezifischen Wärmekapazität bestimmt. Diese hängen wesentlich von den Kluft- und Porenfüllungen (Wasser, Luft) ab, welche eine geringere Wärmeleitfähigkeit als feste Mineralbestandteile aufweisen. Es muss jedoch darauf hingewiesen werden, dass vor allem in klüftigen Gesteinen ein wesentlicher Teil des Wärmetransports durch Konvektion erfolgt. Bei Thermal Response-Tests in Österreich wurden die Kennwerte der Tabelle 1 festgestellt.

Im Jahr 2009 wurden in Oberösterreich die Wärmeleitfähigkeiten, die spezifischen Wärmekapazitäten und teilweise die Dichten der wesentlichen Fest- und Lockergesteine teils in situ, teils im Labor bestimmt (Bertha, Niederbrucker 2009). Die Ergebnisse sind in Tabelle 3 zusammengefasst.

Die hier festgestellten Wärmeleitfähigkeiten liegen wesentlich unter den Literaturwerten und den Betriebserfahrungen über Entzugsleistungen von EWS. So liegen etwa die empfohlene Rechenwerte der SIA 384/6 für Schweizer Molassegesteine zwischen 2,3 und 2,9W/m.K und somit wesentlich über den gemessenen Medianwerten von 1,42 W/m.K für Schlier und von 1,49 W/m.K für die Sande der Molasse. Lediglich die Wärmeleitfähigkeiten der quartären Kiese wurden bei der Berechnung von Thermalfahnen bisher mit $0,5 \mathrm{~W} / \mathrm{m} . \mathrm{K}$ im ungesättigten Bereich wesentlich zu tief angesetzt. Es erscheint ein Ansatz von 1,5 W/m.K entsprechend dem festgestellten Medianwert zulässig, was zu realistischeren Abschätzungen der Thermalfahnen und zu kleineren Ausdehnungen der Temperaturanomalien führt.

\section{Aufzeichnung der Betriebsdaten} 2008/09 von EWS in Oberösterreich

Im Dezember 2008 wurden ca. 150 Wärmepumpenbetreibern, bei denen die wasserrechtliche Überprüfung anstand, Formulare mit der Bitte zugesandt, bis Mitte April 2009 möglichst täglich die min. Vorund Rücklauftemperaturen der EWS sowie einmal wöchentlich die Betriebsstunden der WP aufzuzeichnen. Es wurden die von den Wärmepumpenaggregaten digital angezeigten Werte der Betriebsdaten aufgezeichnet. Eine Überprüfung der Messgenauigkeit der Temperaturfühler erfolgte nicht. Im Frühjahr/Sommer 2009 wurden die Formulare eingesammelt und ausgewertet. Die technischen Daten der Anla-

\begin{tabular}{|c|c|c|c|c|c|c|c|}
\hline & $\begin{array}{l}\text { beheizte } \\
\text { Wohnfläche } \\
{\left[\mathrm{m}^{2}\right]}\end{array}$ & $\begin{array}{l}\text { spez. } \\
\text { Heizleistung } \\
{\left[\mathrm{W} / \mathrm{m}^{2}\right]}\end{array}$ & $\begin{array}{l}\text { Energie- } \\
\text { kennzahl } \\
{\left[\mathrm{kWh} / \mathrm{m}^{2} . \mathrm{a}\right]}\end{array}$ & $\begin{array}{l}\text { Jahresbetriebs- } \\
\text { stunden WP } \\
\text { [h/a] }\end{array}$ & $\begin{array}{l}\text { Einwohner } \\
{[E]}\end{array}$ & $\begin{array}{l}\text { spez. Energie- } \\
\text { bedarf WW } \\
{\left[\mathrm{kWh} /\left(\mathrm{E}^{\star} \mathrm{d}\right)\right]}\end{array}$ & $\begin{array}{l}\text { davon mit WP } \\
{\left[k_{W h} /\left(E^{\star} d\right)\right]}\end{array}$ \\
\hline Median & 162 & 63 & 114 & 2016 & 4 & 3,5 & 3 \\
\hline Anzahl & 28 & 28 & 28 & 28 & 25 & 25 & 25 \\
\hline Max. & 375 & 101 & 192 & 3733 & 5 & 7 & 7 \\
\hline Min & 100 & 28 & 37 & 790 & 1 & 2 & 1 \\
\hline
\end{tabular}




\begin{tabular}{|c|c|c|c|c|}
\hline Energiebedarf Heizen und Warmwasserbereitung & [kWh/Jahr] & 24.872 & & \\
\hline Jahresbetriebsstunden WP & {$[\mathrm{h}]$} & 2.463 & & \\
\hline Gesteinstyp & & Schlier & & \\
\hline Wärmeleitfähigkeit & {$[\mathrm{W} / \mathrm{m} . \mathrm{K}]$} & 2,95 & & \\
\hline spez. Wärmekapazität & {$\left[\mathrm{MJ} /\left(\mathrm{m}^{3} \cdot \mathrm{K}\right)\right]$} & & & \\
\hline Sondenrohre & & Duplex 32 & & \\
\hline spez. Entzugsleistung & [W/Bohrmeter] & 42,5 & aus SIA 384/6, Fig. 11 & \\
\hline Anzahl der EWS & [Stk.] & 2 & & \\
\hline Sondenabstand & {$[\mathrm{m}]$} & 15 & & \\
\hline Korrekturfaktor (Zuschlag) für Simplex-EWS & {$[-]$} & & aus SIA 384/6, Fig.13 & \\
\hline $\begin{array}{l}\text { Korrekturfaktor (Zuschlag) Jahresbetriebsstunden, } \\
\text { Sondenabstand }\end{array}$ & {$[-]$} & 0,16 & \multicolumn{2}{|c|}{ aus SIA 384/6, Fig. 15 bis 20} \\
\hline Sondenlängen & L EWS 1 [m] & 99,6 & & \\
\hline Höhe über Meer & [müA.] & 400 & & \\
\hline Bodenoberflächentemperatur Heizen & {$\left[{ }^{\circ} \mathrm{C}\right]$} & 10,6 & aus SIA 384/6, Fig. 10 & \\
\hline Temperaturgradient & {$[\mathrm{K} / \mathrm{m}]$} & 0,030 & & \\
\hline mittl. Auslegetemperatur EWS & {$\left[{ }^{\circ} \mathrm{C}\right]$} & 0 & $1 \mathrm{~K}$ unter Messung & \\
\hline Bodentemperaturdifferenz & {$[\mathrm{K}]$} & 12,09 & & \\
\hline Sondenlänge mit Korrekturfaktor Bodentemperatur & L EWS 2 [m] & 94,7 & & \\
\hline Bodentemperaturdifferenz & {$[\mathrm{K}]$} & 12,02 & & \\
\hline Sondenlänge mit Korrekturfaktor Bodentemperatur & L EWS 3 [m] & 95,3 & & \\
\hline Bodentemperaturdifferenz & {$[\mathrm{K}]$} & 12,03 & & \\
\hline $\begin{array}{l}\text { Sondenlänge mit Korrekturfaktor } \\
\text { Bodentemperatur }\end{array}$ & L EWS 4 [m] & 95,2 & ausgeführt 2 * $95 \mathrm{~m}$ & \\
\hline spez. Wärmeentzug mit Korrekturfaktoren & [W/Bohrmeter] & 38,33 & & \\
\hline spez. Entzugsarbeit * & {$\left[\mathrm{kWh} /\left(\mathrm{m}^{*} \mathrm{Jahr}\right)\right]$} & 130,58 & $80-100 \mathrm{kWh} /\left(\mathrm{m}^{*} \mathrm{Jahr}\right)$ & \\
\hline
\end{tabular}

Abb. 5: Nachrechnung einer Sondenanlage.

gen wurden aus dem Wasserrechtsakt entnommen. Anschließend wurden mit jedem Anlagenbetreiber persönliche Gespräche geführt und ergänzende Anlagendaten erhoben.

Im Sommer 2009 wurden die Betriebsstundenzählerstände der WP nacherhoben, um die Betriebsstunden für die Warmwasserbereitung abschätzen und die Hochrechnung der Jahresbetriebsstunden Heizen genauer durchführen zu können. Ebenso wurden teilweise vorhandene Zählerstände der Betriebsstundenzähler Warmwasserbereitung und der Wärmemengenzähler nacherhoben. Bei Anlagen mit Drehzahlregelung wurden zur Ermittlung der Jahresbetriebsstunden auch die Zählerstände der Stromzähler und die Leistungen der miterfassten Umwälzpumpen nacherhoben.

\section{Auswertung der Betriebsdaten $2008 / 09$ von EWS in Oberösterreich}

Die ab Dezember/Jänner aufgezeichneten Betriebsstunden „Heizen“ wurden mit den Heizgradtagen (HGT) der Luftgütemessstelle Braunau auf Jahresbetriebsstunden hochgerechnet. Anlagen mit nicht ausreichender Datendichte oder Datenqualität wurden ausgeschieden. Bis Oktober 2009 konnten die Betriebsdaten von 28 Anlagen ausgewertet werden.
Im Zeitraum September 2008 bis Juni 2009 wiesen die Messtelle Steyr 3.423 HGT und die Messtelle Braunau 3.362 HGT auf. Die HGT des Jahres 2008/09 lagen damit im unteren Bereich der Schwankungsbreite. Die HGT des Jahres 1996 wurden bei weitem nicht erreicht.

Die Energiekennzahlen der Gebäude wurden zwischen 37 und $192 \mathrm{kWh} / \mathrm{m}^{2}$,a festgestellt, der Medianwert lag bei $115 \mathrm{kWh} / \mathrm{m}^{2}$.a. Der Energieverbrauch für Heizen wurde bei der Bemessung oft stark unterschätzt. Daraus resultierten Unterdimensionierungen und hohe Jahresbetriebsstunden der WP von bis $\mathrm{zu}$ $3.733 \mathrm{~h} / \mathrm{a}$ (siehe Tab. 4)

Für Bemessung der EWS relevante Betriebsdaten

\begin{tabular}{|c|c|c|c|c|c|c|c|c|}
\hline $\begin{array}{l}\text { Jahres } \\
\text { betriebs- } \\
\text { stunden } \\
\text { [h/a] }\end{array}$ & $\begin{array}{l}\text { Anzahl } \\
\text { EWS } \\
\text { [Stk.] }\end{array}$ & $\begin{array}{l}\text { Sonden } \\
\text { abstand } \\
\text { [m] }\end{array}$ & $\begin{array}{l}\text { Länge } \\
\text { je EWS } \\
\text { [m] }\end{array}$ & $\begin{array}{l}\text { spez. } \\
\text { Entzugs- } \\
\text { leistung } \\
{[\mathrm{W} / \mathrm{m}]}\end{array}$ & $\begin{array}{l}\text { Wärme- } \\
\text { leitfähig- } \\
\text { keit } \\
\text { [W/m.K] }\end{array}$ & $\begin{array}{l}\text { min. } \\
\text { Vorlauf } \\
\text { EWS } \\
{\left[{ }^{\circ} \mathrm{C}\right]}\end{array}$ & $\begin{array}{l}\min . \\
\text { Rücklauf } \\
\text { EWS } \\
{\left[{ }^{\circ} \mathrm{C}\right]}\end{array}$ & $\begin{array}{l}\text { mittl. Aus- } \\
\text { legungs- } \\
\text { temperatur } \\
\text { EWS } \\
{\left[{ }^{\circ} \mathrm{C}\right]}\end{array}$ \\
\hline 2016 & 2 & 7 & 100 & 46 & 2,8 & $-3,0$ & 0,0 & $-2,3$ \\
\hline 28 & 28 & 21 & 28 & 28 & 26,0 & 27,0 & 27,0 & 28,0 \\
\hline 3733 & 4 & 22 & 200 & 62 & 4,7 & 3,0 & 6,8 & 3,5 \\
\hline 790 & 1 & 4,2 & 62 & 31 & 1,8 & $-6,0$ & $-5,0$ & $-6,5$ \\
\hline
\end{tabular}


TABELLE 6

Wärmeleitfähigkeiten der Oö Molasse

\begin{tabular}{llc} 
PLZ & Ort & Wärmeleitfähigkeit [W/m.K] \\
\hline 4715 & Taufkirchen a.d. Trattnach & 2,5 \\
\hline 4980 & Antiesenhofen & 3,3 \\
\hline 4753 & Taiskirchen & 2,8 \\
\hline 4910 & Ried i.I. & 2,5 \\
\hline 4926 & St. Marienkirchen a.H. & 2,4 \\
\hline 4754 & Andrichsfurt & 2,6 \\
\hline 4926 & St. Marienkirchen a.H. & 2,5 \\
\hline 4970 & Eitzing & 1,8 \\
\hline 4921 & Hohenzell & 2,2 \\
\hline 4921 & Hohenzell & 2,3 \\
\hline 4910 & Ried i.I. & 1,8 \\
\hline 4972 & Utzenaich & 2,5 \\
\hline 4910 & Ried i.I. & 2,2 \\
\hline 4910 & Ried i.I. & 2,7 \\
\hline 4751 & Dorf a.d.Pram & 3,0 \\
\hline & Median & $\mathbf{2 , 5}$ \\
\hline & Anzahl & 15,0 \\
\hline & Max. & 3,3 \\
\hline & Min. & 1,8 \\
\hline
\end{tabular}

TABELLE 7

Wärmeleitfähigkeiten der Böhmischen Masse des OÖ Mühlviertels

\begin{tabular}{llc} 
PLZ & Ort & Wärmeleitfähigkeit [W/m.K] \\
\hline 4232 & Hagenberg & 3,5 \\
\hline 4363 & Pabneukirchen & 4,5 \\
\hline 4160 & Aigen i.M. & 4,7 \\
\hline 4161 & Ulrichsberg & 3,9 \\
\hline 4209 & Engerwitzdorf & 2,3 \\
\hline 4221 & Steyregg & 4,5 \\
\hline 4201 & Eidenberg & 4,7 \\
\hline 4209 & Engerwitzdorf & 3,8 \\
\hline 4221 & Steyregg & 2,8 \\
\hline 4209 & Gallneukirchen & 3,0 \\
\hline 4040 & Altenberg & 4,3 \\
\hline 4040 & Lichtenberg & 3,8 \\
\hline & Median & 3,9 \\
\hline & Anzahl & 12,0 \\
\hline & Max. & 4,7 \\
\hline & Min. & 2,3 \\
\hline
\end{tabular}

Der spez. Energiebedarf für Warmwasserbereitung wurde zwischen 2 und $7 \mathrm{kWh} / \mathrm{E}$.d festgestellt, der Medianwert lag bei 3,5 kWh/E,d. Da teilweise im Mai und Juni 2009 noch geheizt wurde und diese Betriebsstunden großteils der Warmwasserbereitung zugerechnet wurden, liegen diese Daten auf der sicheren Seite. Der
Maximalwert von $7 \mathrm{kWh} / \mathrm{E}$, d trat bei einem Einpersonenhaushalt auf, wo die Boilerverluste wesentlich stärker zu gewichten sind.

Die Nachrechnung der EWS erfolgte mit der MS-Excel ${ }^{\mathrm{TM}}$ Rechentabelle des ÖWAV-Regelblattes 207, (www.oewav.at im Bereich „Download“) mit den festge- stellten Betriebsdaten. Die mittlere abgelesene minimale Soletemperatur wurde um $1 \mathrm{~K}$ abgemindert als mittlere Auslegungstemperatur eingetragen. Die spez. Entzugsleistung und die Korrekturfaktoren wurden solange variiert, bis die errechnete Sondenlänge mit der ausgeführten Sondenlänge übereinstimmte.

Die Ergebnisse sind in Tabelle 5 dargestellt.

Die spez. Entzugsleistungen der EWS wurden zwischen 31 und $62 \mathrm{~W} / \mathrm{m}$ festgestellt, der Medianwert lag bei $47 \mathrm{~W} / \mathrm{m}$. Die festgestellten Wärmeleitfähigkeiten des Untergrunds sind in Tabelle 6 dargestellt.

Der Medianwert der festgestellten Wärmeleitfähigkeit der oö Molasse liegt etwas über den empfohlenen Rechenwerten des ÖWAV-Regelblattes 207 und deckt sich gut mit den empfohlenen Rechenwerten der SIA 386/4. Die höchsten Werte können auf nicht berücksichtigte thermische Anomalien oder einen großen Grundwassereinfluss zurückzuführen sein. Für die Bemessung von Anlagen soll maximal der Medianwert angesetzt werden. Auffallend ist die im Bereich Ried i. I./Hohenzell kleinräumig stark variierende Wärmeleitfähigkeit von 1,8 bis $2,7 \mathrm{~W} / \mathrm{m}$.K, welche vor allem auf unterschiedliche Grundwassereinflüsse zurückzuführen sein dürfte.

Der Medianwert der festgestellten Wärmeleitfähigkeit der Böhmischen Masse liegt über den empfohlenen Rechenwerten des ÖWAV-Regelblattes 207 und der SIA 384/6. Es ist möglich, dass nicht die Tiefstwerte der Soletemperaturen im Vorund Rücklauf der EWS aufgezeichnet wurden, woraus bei der Rückrechnung höhere Wärmeleitfähigkeiten resultieren. Für die Bemessung von Anlagen sollten weiter die Literaturwerte des ÖWAV-Regelblatts 207 verwendet werden, da die Daten noch zu wenig abgesichert sind.

Der Medianwert der mittleren abgelesenen minimalen Soletemperaturen lag bei $-1,5^{\circ} \mathrm{C}$. Die mittleren Auslegungstemperaturen der EWS wurden zwischen $-6,5^{\circ} \mathrm{C}$ und $+3,5^{\circ} \mathrm{C}$ festgestellt, der Medianwert lag bei $-2,5^{\circ} \mathrm{C}$. Dabei wurden die aufgezeichneten Temperaturen im Regelfall um $1 \mathrm{~K}$ abgesenkt (Berücksichtigung weiterer Betriebsjahre, Unsicherheit, ob min. Werte gemessen wurden, kein extremer Winter). Die Ursachen für die Unterschreitung der min. zulässigen mittleren Auslegungstemperatur von $-1,5^{\circ} \mathrm{C}$ sind:

- höhere Heizlast als berechnet/abgeschätzt

- Entzugsleistung der EWS überschätzt

- Korrekturfaktoren für Simplex-Sonden, geringe Sondenabstände und erhöhte 
Betriebsstunden nicht berücksichtigt

- Energiebedarf der Warmwasserbereitung unterschätzt

Simplex 40-Sonden weisen gegenüber Duplex 32-Sonden bei einer Spreize von $3 \mathrm{~K}$ eine um 13 bis $19 \%$ geringere Entzugsleistung auf, was bei der Bemessung von 4 ausgewerteten Simplex-Sonden nicht berücksichtigt wurde. Es wird deshalb von der Errichtung von SimplexSonden abgeraten. Die SIA 384/6 enthält ein Diagramm über den für Simplex-Sonden erforderlichen Zuschlag in Abhängigkeit von der Wärmeleitfähigkeit des Untergrunds.

Unterhalb einer minimalen EWS-Temperatur von -3 bis $-5^{\circ} \mathrm{C}$ ist gemäß SIA 384/6 infolge Eisbildung mit Schäden an den EWS oder den berührenden Bauteilen zu rechnen. Für EWS, welche diesen Temperaturbereich erreichen oder unterschreiten, sind zur Vermeidung von Schäden und zur Sicherstellung der zu erwartenden Nutzungsdauer von mind. 50 Jahren Sanierungsmaßnahmen zu setzen. Diese sind beispielhaft:

- Verminderung des Wärmeentzugs aus der Sonde: Verbesserung der Wärme- dämmung des Gebäudes, ergänzende Solaranlage zur Warmwasserbereitung, Zusatzheizung

- Thermische Regeneration durch Kühlen,

- Errichtung einer zusätzlichen EWS

- Optimierung der Hydraulik der EWS

\section{Ausblick}

Die Betriebsdatenaufzeichnungen von EWS und die Auswertungen werden für 2009/10 verstärkt fortgeführt. Aus den bisherigen Erfahrungen wurden Betreiber mit Interesse an den Aufzeichnungen ersucht, im Winter 2009/10 möglichst einmal täglich die Temperaturen des Vorlaufs und des Rücklaufs der EWS bei Betrieb der Wärmepumpe in ein Formular einzutragen. Weiters wurden diese Betreiber ersucht, einmal monatlich die Zählerstände der Wärmepumpe, des Wärmemengenzählers und der Stromzähler der Wärmepumpe einzutragen. Bei der Auswertung ist der Plausibilitätsprüfung der Daten höchste Priorität einzuräumen.

Eine systematische Betriebsdatenerfassung, welche jede Anlage zu einer Bemessungsanlage macht, erscheint nur

\section{LITERATUR}

ÖWAV Regelblatt 207 (2009) Thermische Nutzung des Grundwassers und des Untergrunds Heizen und Kühlen; ÖWAV Regelblatt 207 Norm SIA 384/6 (2009) Erdwärmesonden Entwurf Norm SIA 384/6 (2008) Erdwärmesonden Niederbrucker R, Steinbacher N (2007) Eignungsuntersuchung von Verpressmaterialien für nungsuntersuchung von Verpressmaterialien für Erdwärmesonden, Technischer Endbericht; Herload: www.land-oberoesterreich.gv.at, weiter unter $>$ Themen $>$ Umwelt $>$. Wasser $>$ Grundwasser $>$ Thermische Nutzung Bertha E, Niederbrucker R (2009) Bestimmung der thermischen Parameter von repräsentativen
Gesteinen Oberösterreichs, Herausgeber: Amt GEO-Pot Oberflächennahes Geothermie Potenzial Österreich, Präsentation im Rahmen der ÖWAV-Sitzung vom 01-10 bis 02-10-2009, Geologische Bundesanstalt

Hydrographisches Jahrbuch von Österreich 2005 (2008) 113. Band, Hydrografischer Dienst in Österreich

Danninger E et al. (2008) Überwachungsbericht des oö Luftmessnetzes, Jahresbericht 2007.

Download: www.land-oberoesterreich.gv.at, weiter unter $>$ Themen $>$ Umwelt der Oö Landesregierung

Götzl G (2009) Geologische Bundesanstalt: möglich, wenn täglich die min. Vorlaufund Rücklauftemperaturen des Wärmeträgermediums bei Anlagenbetrieb und periodisch die Betriebsstunden registrierend erfasst werden und die Daten in einem gängigen Format ausgelesen werden können. Die Vorlauf- und Rücklauftemperaturen des Wärmeträgermediums müssten dazu bei Anlagenbetrieb z. B. viertelstündlich registriert und z.B. der Minimalwert des jeweiligen Tages gespeichert werden, sodass Jahrestemperaturganglinien zur Verfügung stehen. Die Wärmepumpenhersteller werden deshalb dringend ersucht, europaweit standardisierte Speichermodule serienmäßig in die Anlagen zu implementieren, was mit den heute zur Verfügung stehenden Speichermedien leicht realisierbar sein müsste. Die Vorteile liegen auf der Hand:

- Jede EWS wird zur Bemessungsanlage

- die Jahresganglinie der Grundwassertemperatur bei Grundwasserwärmepumpenanlagen wird dokumentiert

- die Messdaten erleichtern die Optimierung des Wärmepumpenbetriebs

- genaue Betriebsdaten minimieren Konflikte mit benachbarten Nutzungen.

Die Wärmeleitfähigkeitsdaten der Thermal Response Tests sowie der Betriebsdatenauswertungen werden in einem Wärmeleitfähigkeitskataster dargestellt, welcher unter doris.ooe.gv.at > Fachinformation > Wasser \& Geologie veröffentlicht und zunehmend verdichtet wird.

Korrespondenz:

DI Johann Aschauer

Amt der Oö Landesregierung

Direktion Umwelt und Wasserwirtschaft

Abteilung Grund- und Trinkwasserwirtschaft

4021 Linz, Kärntnerstraße 10-12

E-Mail: johann.aschauer@ooe.gv.at 